\title{
Relationship of splanchnic blood flow and portal venous resistance to elevated portal pressure in the $\operatorname{dog} 1$
}

\author{
C. L. Witte, G. R. TOBin, D. S. ClARK, AND M. H. Witte 2 \\ From the Department of Surgery, University of Arizona College of Medicine, Tucson, Arizona, U.S.A.
}

SUMMARY To determine the relationship of splanchnic blood flow and portal venous resistance to elevated portal pressure, in situ perfusion of the splanchnic circuit was carried out in 12 freshly killed dogs at varying perfusion rates and degrees of acute and chronic portal vein constriction. In six normal dogs before being killed, portal pressure averaged $8.8 \pm 1 \cdot 2 \mathrm{mmHg}$ and portal flow $658 \pm 81$ $\mathrm{ml} / \mathrm{min}$. In the absence of portal vein constriction, increasing splanchnic perfusion to $1800 \mathrm{ml} / \mathrm{min}$, minimally raised portal pressure $(12.8 \pm 1.5 \mathrm{mmHg})$. With progressive acute constriction of the portal vein, however, comparable perfusion rates lead to progressively higher portal pressure levels and with $>90 \%$ constriction, portal pressure was $>30 \mathrm{mmHg}$ with minimal elevation in splanchnic flow rate. In six other dogs before being killed but live to nine weeks after placement of an ameroid constrictor on the portal vein, portal pressure averaged $13.6 \pm 1.4 \mathrm{mmHg}$ or slightly higher than in normal dogs ( $\mathrm{P}>0.02)$. Mesenteric venography and necropsy findings uniformly demonstrated $90 \%$ occlusion of the portal vein with extensive portasystemic collateralization. With increased perfusion of the splanchnic bed, portal pressure rose rapidly to $\sim 35 \mathrm{mmHg}$ with a flow rate of $1800 \mathrm{ml} / \mathrm{min}$.

These data suggest that, in disorders where resistance to transhepatic portal flow is marked, a small increment in splanchnic blood flow, which normally exerts little or no influence on portal pressure, promotes profound portal hypertension and may account for spontaneous 'rupture' of oesophageal varices. On the other hand, where resistance to transhepatic portal flow is mild but splanchnic blood flow is markedly hyperdynamic, reducing inflow may be sufficient to ameliorate extreme portal hypertension and its sequelae.

Failure to reproduce in experimental animals the major clinical manifestations of portal hypertension has been a significant factor limiting comprehension of the pathophysiology of this syndrome. Thus, sustained portal pressure elevation in the range of 25 $\mathrm{mmHg}$, bleeding from oesophageal varices, and ascitic fluid low in protein content, findings commonly observed in patients with hepatic cirrhosis, do not usually accompany experimental constriction of the portal or hepatic veins. Nonetheless, each of these serious clinical complications is generally conceded to be somehow a direct result of increased portal vascular resistance with portal hypertension. Pressure in the portal venous system, however, depends

'Supported by USPHS Grants HL 13341 and HL 13390. ${ }^{2}$ Currently the recipient of USPHS Career Research Development Award (HL-19605).

Received for publication 14 November 1975 not only upon resistance to portal flow through the liver and through portasystemic collaterals bypassing the liver, but also upon the volume of splanchnic blood feeding into the portal system from the digestive tract and spleen (Bradley, 1963). Not surprisingly, therefore, it has been suggested that hyperdynamic splanchnic blood flow alone (Womack and Peters, 1961) or in combination with increased resistance to transhepatic portal flow (Witte and Witte, 1975) accounts for the varied clinical presentations of patients with the portal hypertension syndrome.

To provide more quantitative data on the interrelationships between splanchnic blood flow and portal venous resistance on the one hand and portal pressure on the other, portal pressure was serially measured in freshly killed dogs during in situ stepwise increments in splanchnic perfusion with varying degrees of portal venous constriction. 


\section{Methods}

In 12 mongrel dogs $(14-29 \mathrm{~kg})$, fasted for 12 hours except for water, and lightly anaesthetized with intravenous pentobarbital $(25 \mathrm{mg} / \mathrm{kg})$, portal venous flow and/or portal pressure were determined (see groups 1 and 2 , vide infra). After these measurements, heparin sodium $(2 \mathrm{mg} / \mathrm{kg})$ was administered intravenously, fatal exsanguination via the femoral artery was induced, and the blood set aside for later reinfusion. The abdominal aorta was cannulated for perfusion of the coeliac-superior mesenteric arteries and the aorta ligated at the level of the diaphragm and just above the renal arteries. The thoracic inferior vena cava was cannulated via the right atrium for collection of hepatic venous drainage and the abdominal vena cava ligated above the renal veins. The vena cava catheter was connected via a reservoir (filled with heparinized blood just removed during exsanguination) to a roller pump for reinfusion via the aortic cannula under controlled flow rates(Fig.1). A jejunal mesenteric vein previously cannulated for premortem measurement of portal pressure was used to monitor portal pressure continuously on a direct writing recorder. Perfusion time at each flow rate was approximately five minutes.

\section{GROUP 1 (six dogs)}

Immediately before killing each dog, portal venous flow was measured using a non-cannulating square-wave electromagnetic flowmeter (Carolina Electronics) and the in situ perfusion model as outlined above was then instituted. Starting at a flow rate of $300 \mathrm{ml} / \mathrm{min}$, the arterial inflow rate was increased in step-wise fashion up to $1800 \mathrm{ml} / \mathrm{min}$ or to a portal pressure $>35 \mathrm{mmHg}$. At this point, infusion was stopped, the portal vein constricted to approximately $75 \%$ of its original diameter, and arterial inflow restarted and increased in a stepwise fashion as before. This manoeuvre was then repeated with the portal vein progressively narrowed to $50 \%$, $25 \%, 10 \%$, and $0 \%$ of the original diameter, and the effect of arterial inflow on portal pressure continuously monitored.

\section{GROUP 2 (six dogs)}

Five to nine weeks before final studies, an ameroid constrictor (casein hydrolysate) was placed around the portal vein under pentobarbital anaesthesia, and the dogs allowed to recover. At the time of final study, the portal pressure was measured via a jejunal mesenteric vein catheter, the dog killed by exsanguination, the regional perfusion model as described above instituted, and a 'single-run' of splanchnic blood flow measurements in relation to portal pressure levels determined. In five dogs portal venography

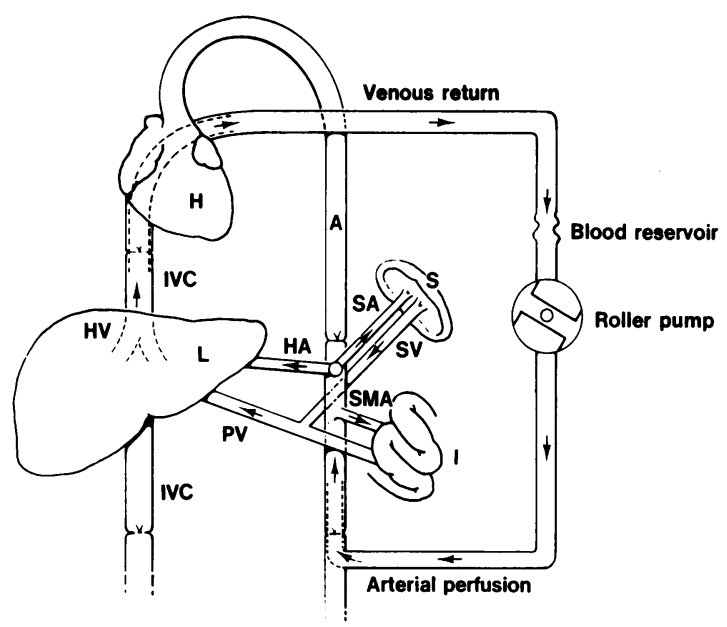

Fig. 1 Schematic diagram of in situ preparation of splanchnic perfusion in freshly killed dogs. During experimentation splanchnic blood flow was sequentially increased with varying degrees of portal venous constriction and the effect on portal pressure determined. (See Methods section for technical operative details.) $H$ : heart. L: liver. S: spleen. I: intestines. A: aorta. $S A$ : splenic artery. $S M A$ : superior mesenteric artery. $S V$ : splenic vein. $P V$ : portal vein. IVC: inferior vena cava. $H A$ : hepatic artery. $H V$ : hepatic vein.

was also performed via the jejunal mesenteric catheter, and in each dog the portal vein was examined at the end of the experiment to confirm the degree of portal vein constriction.

\section{Results}

\section{GROUP 1}

Premortem portal venous flow averaged $658 \pm 81$ $\mathrm{ml} / \mathrm{min}$ and portal pressure $8.8 \pm 1.2 \mathrm{mmHg}$. After death and perfusion, but without portal vein constriction, progressive increase in arterial flow to 1800 $\mathrm{ml} / \mathrm{min}$ raised portal pressure minimally to $12.8 \pm$ $1.5 \mathrm{mmHg}^{1}$ (Fig. 2). On the other hand, with incremental constriction of the portal vein, comparable perfusion rates led to progressively higher portal pressure levels. With high grade portal vein obstruction $(>90 \%)$, portal pressure attained levels $>30$ $\mathrm{mmHg}$ with minimal elevations in splanchnic flow rate (Fig. 2).

\section{GROUP 2}

Five to nine weeks after placement of an ameroid constrictor on the portal vein, portal pressure averaged $13.6 \pm 1.4 \mathrm{mmHg}$ or slightly higher than

${ }^{1}$ Portal pressure levels recorded for each perfusion period had been stable for more than four minutes. 


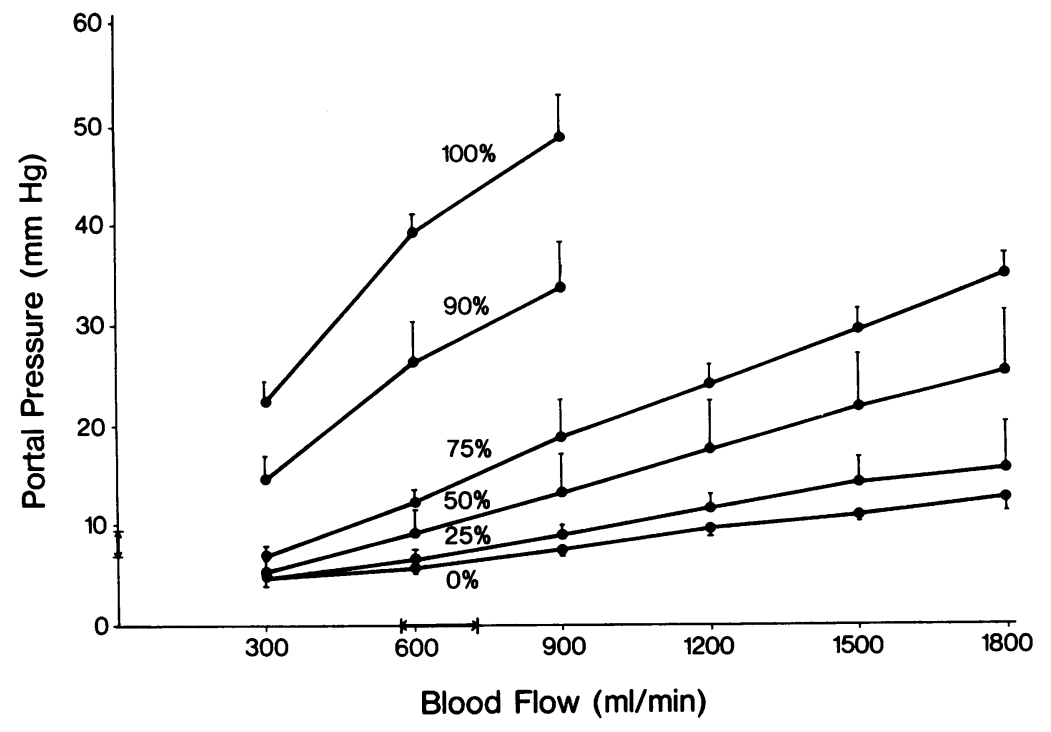

Fig. 2 Relationship of splanchnic blood flow and portal venous resistance to portal pressure (mean \pm $S E)$ during in situ perfusion of six freshly killed dogs (Fig. 1). With progressive constriction of the portal vein, a progressively steeper rise in portal pressure occurred with increasing splanchnic blood flow. The arrowed areas along the abscissa and ordinate represent the range of premortem values of portal blood flow and portal pressure in these dogs.

dogs in group 1 ( $\mathrm{P}>0.02)$. Portal venography and final necropsy findings documented near total obstruction of the portal vein and extensive portasystemic collateralization in each dog (Fig. 3). After perfusion of the splanchnic bed, portal pressure rose rapidly ${ }^{1}$ and reached $>35 \mathrm{mmHg}$ at a flow rate of $1800 \mathrm{ml} / \mathrm{min}$ (Fig. 4).

\section{Discussion}

While increased resistance to portal blood flow is generally conceded to be a hallmark of portal hypertension, the varied clinical presentations of this syndrome and their relation to underlying splanchnic haemodynamic abnormalities remain puzzling. Some patients present with massive ascites, repeated haemorrhage from oesophageal varices and severe hypersplenism, whereas others with equivalent or even higher portal pressure readings may be symptom-free. Previous studies have indicated that, in many of these patients, a generalized hyperdynamic circulatory state coexists with portal hypertension(Kowalski and Abelmann, 1953; Murray et al., 1958). In experimental animals, however, and probably in man, disturbances characterized solely by a hyperdynamic circulation are not usually accompanied by marked elevation in portal pressure ( $>25 \mathrm{mmHg}$ ), ascites, or bleeding oesophageal varices (Witte et al., 1974). On the other hand, passive congestion from increased resistance to transhepatic portal flow is also not a

${ }^{1}$ Portal pressure levels recorded for each perfusion period had been stable for more than four minutes.

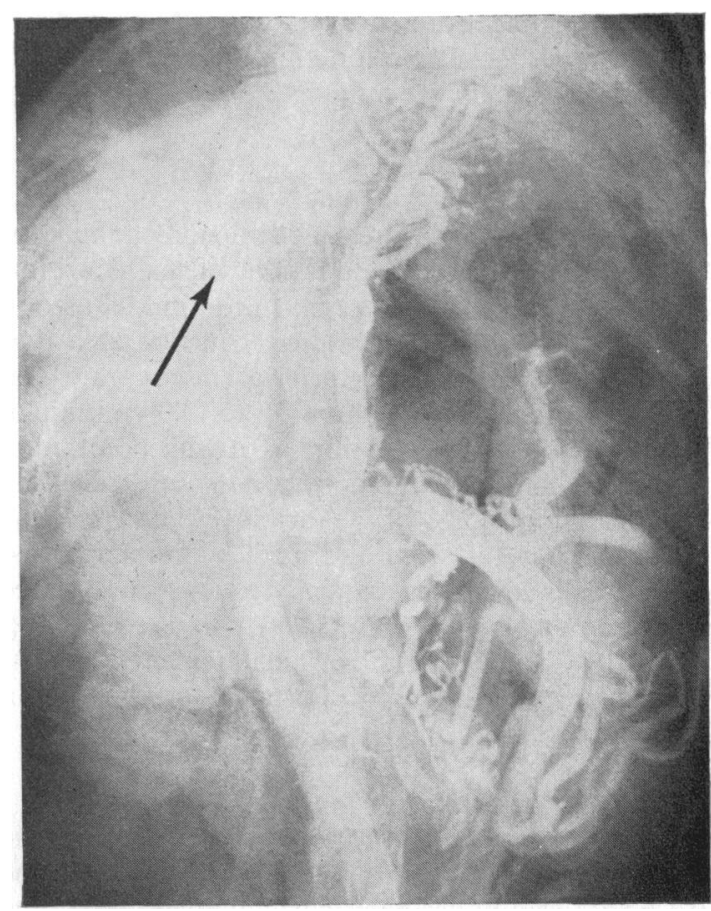

Fig. 3 Characteristic $x$-ray appearance of portal vascular system in a dog six weeks after placement of an ameroid constrictor (casein hydrolysate) on the portal vein.

Despite nearly $90 \%$ occlusion of the portal vein (arrow), portal pressure level in this dog measured only $15 \mathrm{mmHg}$. The portal system was visualized by cannulation of a jejunal mesenteric vein and intravenous injection of $70 \%$ Renografin. 


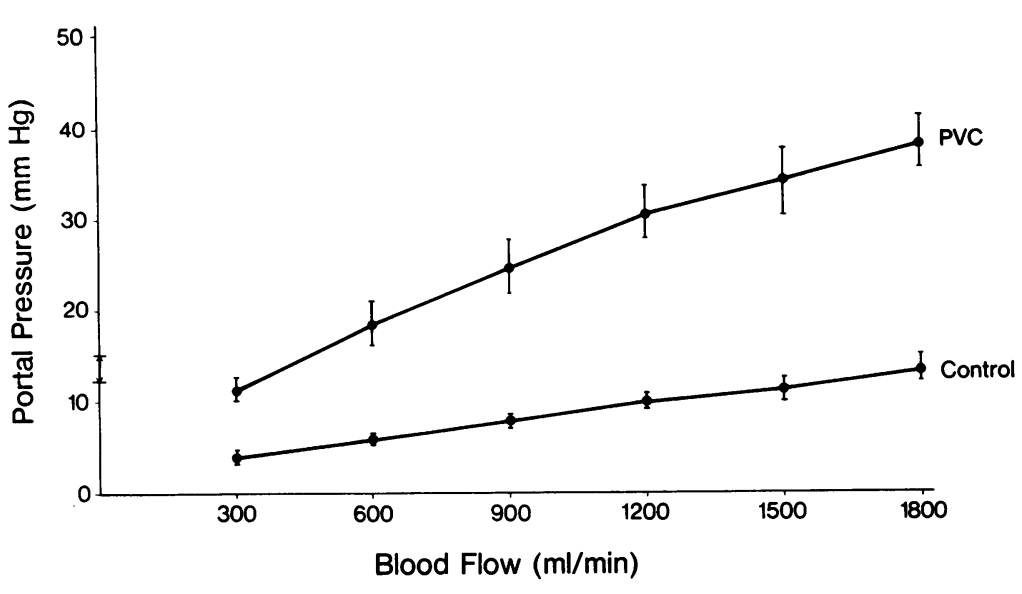

Fig. 4 Relationship of splanchnic blood flow and portal venous resistance to portal pressure (mean $\pm S E$ ) during in situ perfusion of six freshly killed dogs, five to nine weeks after placement of an ameroid constrictor (casein hydrolysate) on the portal vein $(P V C)$. Although portal pressure was only slightly elevated (see range arrowed on ordinate axis) as splanchnic blood flow increased a much sharper rise in portal pressure levels occurred compared with dogs without portal vein constriction (control, taken from Fig. 2, $0 \%$ ).

fully satisfactory explanation of the clinical picture (Witte and Witte, 1974).

Based upon measurements of splanchnic blood flow and portal vascular resistance in patients with hepatic cirrhosis and in dogs with glucagon-stimulated hyperdynamic splanchnic blood flow with and without portal vein constriction, an explanation of portal hypertension has been proposed wherein both haemodynamic mechanisms (increased blood flow and increased resistance to portal flow) working in concert, produce active congestion (Witte et al., 1974). Physiological features of this experimental phenomenon mimic closely the circulatory disturbance observed in human portal hypertension. Furthermore, it has been suggested that determination of the individual haemodynamic patterns in these patients not only leads to a more complete physiological understanding of portal haemodynamics, but also provides the rationale for sounder operative and non-operative treatment of the serious complications of this disorder (Witte et al., 1969; Witte et al., 1972).

Expanding upon earlier work, Zimmon and Kessler (1974) demonstrated a close correlation between splanchnic blood volume and portal pressure. Furthermore, they suggested that the likelihood of complications from portal hypertension was related to the dynamic interplay between these two variables. The experimental haemodynamic studies reported here provide a fuller explanation of this interrelationship by considering splanchnic blood flow rather than the more static and elusive concept of blood volume. Although our studies were performed postmortem, thereby eliminating active metabolic influences on vascular tone, the measurements nonetheless illustrate that similar levels of portal pressure elevation may be obtained by different combinations of splanchnic blood flow and portal vascular resistance. Despite the artificiality of the experimental situation, portal pressure readings at comparable splanchnic flow rates were similar whether recorded immediately before or promptly after the animal's death under conditions of perfusion. Thus, in control states before death, portal pressure levels averaged approximately $9 \mathrm{mmHg}$ and portal flow rate approximately $660 \mathrm{ml} / \mathrm{min}$, while at a similar splanchnic flow rate during perfusion portal pressure was $7 \mathrm{mmHg}$. This difference in portal pressure post-mortem probably reflects a slight diminution in vascular tone and hence diminished resistance in the portal vascular system. Accordingly, while these measurements may require quantitative adjustment to the in vivo state, the data suggest nonetheless that (1) increased splanchnic blood flow in the absence of restriction to transhepatic portal flow does not generate portal hypertension; (2) with progressive increase in portal vascular resistance, a correspondingly smaller increase in splanchnic blood flow is required to generate severe portal hypertension; (3) marked portal hypertension may be generated by markedly increased portal vascular resistance and minimally increased splanchnic blood flow, or by markedly increased splanchnic blood flow and minimally increased portal vascular resistance, or by moderate increases in both factors; (4) chronic severe constriction of the portal vein leads only to mild elevation in portal pressure, but when increased splanchnic blood is superimposed a pronounced rise in portal pressure occurs; (5) diminution in severity of portal hypertension that develops during marked chronic as contrasted with acute portal vein constriction and increased perfusion is probably attributable to por- 
tal venous collateralization in the chronic preparation. Thus, development of these collaterals enhances portal venous capacitance and comparable rates of splanchnic flow lead to a smaller portal pressure elevation.

As with central venous pressure, portal pressure depends upon a whole set of complex rapidly changing blood flow-vascular resistance dynamics, albeit in the splanchnic rather than systemic circuit. Whereas cardiac catheterization has uncovered many of the haemodynamic interrelationships in the cardiac and pulmonary circulations leading to a rational explanation for central venous hypertension, direct measurements and similar correlations in the portal system have been difficult to come by. The studies described here, although performed under less than ideal conditions, provide a reasonable explanation for the wide spectrum of physiological and clinical findings in portal hypertension. For instance, in disorders where resistance to transhepatic portal flow is marked (for example, advanced hepatic cirrhosis or portal vein thrombosis) a small increment in splanchnic blood flow (for example, excessive ingestion of alcohol or hormonal influences) which normally exerts little or no influence on portal pressure, promotes profound portal hypertension. This steep response curve may explain 'spontaneous' rupture of oesophageal varices with haemorrhage in susceptible patients. On the other hand, where resistance to transhepatic portal flow is mild (for example, early hepatic cirrhosis or myeloproliferative disorders) but where splanchnic blood flow is markedly hyperdynamic (for example, massive splenomegaly) reducing inflow may be sufficient to ameliorate portal hypertension and its sequelae. Accordingly, it is anticipated that any single therapeutic approach applied to all patients with the portal hypertension syndrome is not likely to be uniformly successful.

\section{References}

Bradley, S. E. (1963). The hepatic circulation. In Handbook of Physiology, section 2, vol. 2, pp. 1387-1438. American Physiological Society, Washington, D.C.

Kowalski, H. J., and Abelmann, W. H. (1953). The cardiac output at rest and in Laennec's cirrhosis. Journal of Clinical Investigation, 32, 1025-1033.

Murray, J. F., Dawson, A. M., and Sherlock, S. (1958). Circulatory changes in chronic liver disease. American Journal of Medicine, 24, 358-367.

Witte, C. L., and Witte, M. H. (1974). Hepatic circulation. In The Liver: Normal and Abnormal Functions, pp. 11-54. Edited by F. Becker. Dekker: New York.

Witte, C. L., and Witte, M. H. (1975). The circulation in portal hypertension. Yale Journal of Biology and Medicine, 48, 141-155.

Witte, C. L., Witte, M. H., Bair, G., Mobley, W. P., and Morton, D. (1974). Experimental study of hyperdynamic vs. stagnant mesenteric blood flow in portal hypertension. Annals of Surgery, 179, 304-310.

Witte, M. H., Witte, C. L., Cole, W. R., and Kochler, P. R. (1969). Contrasting patterns of ascites formation in hepatic cirrhosis. Journal of the American Medical Association, 208, 1661-1666.

Witte, C. L., Witte, M. H., and Krone, C. L. (1972). Contrasting hemodynamic patterns of portal hypertension. Annals of Surgery, 176, 68-79.

Witte, C. L., Witte, M. H., Renert, W., and Corrigan, J. J. (1974). Splenic circulatory dynamics in congestive splenomegaly. Gastroenterology, 67, 498-505

Womack, N. A., and Peters, R. M. (1961). The significance of splenomegaly in cirrhosis of the liver. Annals of Surgery, 153, 1006-1019.

Zimmon, D. S., and Kessler, R. E. (1974). The portal pressure-blood volume relationship in cirrhosis. Gut, 15, 99101. 